\title{
Percepción del personal de salud sobre un programa de detección oportuna del cáncer cervical
}

\section{Health personnel perceptions on a cervical cancer early detection program}

\section{Percepção do pessoal de saúde sobre um programa de detecção oportuna do câncer cervical}

\author{
C.I. Hernández-Márquez ${ }^{\mathrm{a}^{*}}$, A.A. Salinas-Urbina ${ }^{\mathrm{b}}$ \\ a Jefatura de posgrado. Facultad de Enfermería. Universidad Autónoma del Estado de Morelos. Cuernavaca, Mo- \\ relos, México.

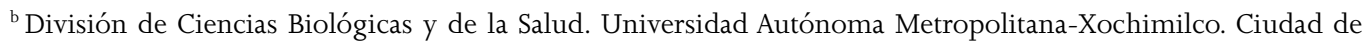 \\ México. México.
}

Recibido: 2 febrero 2017

Aceptado: 29 enero 2018

\section{Resumen}

Objetivo: Conocer la percepción que el personal de salud del primer nivel de atención tiene respecto al programa Detección Oportuna del Cáncer Cervical en México.

Método: Estudio cualitativo, se realizaron 15 entrevistas semi-estructuradas a médicos y enfermeras de tres centros de salud rurales del Estado de Morelos, México. La información se analizó siguiendo los planteamientos de la Teoría Fundamentada.

Resultados: El personal de salud percibe el programa Detección Oportuna del Cáncer Cervical como un programa de salud con fines preventivos, identifica la prueba de citología cervical como el medio para prevenir el cáncer cervicouterino, considera que el objetivo del programa es la obtención de muestras citológicas. La percepción está definida por el cumplimiento de metas, las barreras que se presentan para su operación, la elevada demanda y la escasez de recursos materiales, así como por los sentimientos del personal y sus condiciones de trabajo.

Conclusiones: Es necesario reforzar los planes y programas de estudio de las carreras de enfermería y medicina para que los estudiantes obtengan conocimientos, desarrollen habilidades y adquieran competencias con base en conocimientos científicos, generar el correcto uso de términos médicos, la lectura crítica, la búsqueda de información y el interés por la actualización y crecimiento profesional.

*Autor para correspondencia; correo electrónico: clara.hernandez@uaem.mx (C.I. Hernández-Márquez) 
Palabras clave: Percepción; personal de salud; programas nacionales de salud; detección precoz del cáncer; prevención de enfermedades; México.

\section{Abstract}

Objective: To identify the perceptions which first level of attention health personnel have on the Cervical Cancer Early Detection National Health Program in Mexico. Method:This is a qualitative study which included 15 semi-structured interviews on medicine and nursing personnel of three rural health centers in the State of Morelos, Mexico. Data were analyzed in line with the Grounded Theory.

Results: The health personnel perceives the Cervical Cancer Early Detection Program as a preventive initiative which relies on the cervical cytology as a main procedure to prevent cervical cancer. These perceptions are modulated by issues on the achievement of goals, operation barriers, high demand of the tests, scarcity of resources, and working conditions feelings.

Conclusions: It is important to keep strengthening the nursing and medicine study plans so that students keep developing their knowledge, competencies, and skills to support health initiatives such as the Cervical Cancer Early Detection Program while they develop their scientific interest and professional growth.

Keywords: Perception; health personnel; national health programs; early detection of cancer; disease prevention; Mexico.

\section{Resumo}

Objetivo: Conhecer a percepção que o pessoal de saúde do primeiro nível de atenção tem ao respeito do programa Detecção Oportuna do Câncer Cervical no México.

Método: Estudo qualitativo, realizaram-se 15 entrevistas semiestruturadas a médicos e enfermeiras de três centros de saúde rurais do Estado de Morelos, México. A informação analisou-se seguindo as abordagens da Teoria Fundamentada.

Resultados: O pessoal de saúde percebe o programa Detecção Oportuna do Câncer Cervical como um programa de saúde com fins preventivos, identifica a proba de citologia cervical como o médio para prevenir o câncer de colo do útero, considera que o objetivo do programa é a obtenção de amostras citológicas. A percepção está definida pela realização de metas, as barreiras que se apresentam para sua operação, a elevada demanda e a escassez de recursos materiais, assim como pelos sentimentos do pessoal e suas condições de trabalho.

Conclusões: É necessário reforçar os planos e programas de estudo das carreiras de enfermagem e medicina para que os estudantes obtenham conhecimentos, desenvolvam habilidades e adquiram competências com base em conhecimentos científicos, gerar o correto uso de termos médicos, a leitura crítica, a busca de informação e o interesse pela atualização e crescimento profissional.

Palavras chave: Percepção; pessoal de saúde; programas nacionais de saúde; detecção precoce do câncer; prevenção de doenças; México.

\section{Introducción}

En México, el cáncer cervicouterino ( $\mathrm{CaCu}$ ) se ubica como la segunda causa de muerte por neoplasias en mujeres mayores de 35 años. A pesar de que el Sistema de Salud en México instauró el programa Detección Oportuna del Cáncer Cervical (DOCC) como prioritario para la conservación y mejora de la salud de la población, no se han visto resultados alentadores en la disminución de la mortalidad, especialmente entre la población que vive en áreas rurales, en donde, a nivel nacional, las mujeres que radican en esas regiones tienen un riesgo 6\% mayor de morir por cáncer cervical que aquellas que viven en zonas urbanas, lo que se ve determinado por la existencia de diversas barreras sociales que afectan 
la presencia y gravedad de la enfermedad y disminuyen la sobrevida de las pacientes que la presentan ${ }^{1}$. Una de las principales barreras detectadas a este nivel es el diagnóstico tardío, tarea que compete a los servicios de salud en el primer nivel de atención, en este caso, a través del programa de DOCC. Dicho programa se basa en la realización de la prueba de citología cervical o Papanicolaou (Pap), que constituye el primer eslabón para el diagnóstico de cáncer cervical y es considerada una prueba de detección de rutina para las mujeres de 30 años o más. Consiste en la obtención de una muestra de las células del endocérvix y exocérvix, que posteriormente serán visualizadas a través de un microscopio por un médico especialista (citotecnólogo), en un laboratorio ${ }^{2}$. El objetivo de la prueba es detectar anormalidades morfológicas de las células examinadas que provienen de la descamación de superficies epiteliales, las cuales después de períodos prolongados (10 años o más) podrían convertirse en cáncer ${ }^{3}$ ${ }^{4}$. De acuerdo con las estimaciones de la International Agency for Research on Cancer (IARC), el uso de la citología cervical ha logrado reducir la mortalidad por cáncer de cuello uterino en países desarrolla$\operatorname{dos}^{5}$, pero no en países de América Latina, donde mueren alrededor de 36,000 mujeres cada año por este padecimiento ${ }^{6}$. En México, se estima un total de tres mil 771 defunciones al año por esta causa, lo que representa una tasa de 11.3 defunciones por 100,000 mujeres mayores de 25 años ${ }^{7}$.

Ante los mínimos logros del programa, en México se han realizado investigaciones que intentan identificar las raíces de este padecimiento; así como buscar las causas del fenómeno en el nivel socioeconómico de las mujeres ${ }^{8}$, en la falta de conocimientos del personal de salud ${ }^{9}$, o en las limitadas condiciones de las instituciones donde labora dicho personal ${ }^{10}$. Asimismo, estos temas se han abordado desde la perspectiva cualitativa como se evidencia en la investigación de Chavira ${ }^{11}$ sobre la percepción del personal médico hacia los programas de mejora de la calidad. Sin embargo, en todas las investigaciones realizadas no ha sido posible identificar aspectos que logren explicar los resultados del programa. Por ello, surge el interés por explorar desde la mirada del personal, su percepción respecto a la operatividad del programa DOCC.

De acuerdo a los fundamentos de la teoría de Gestalt ${ }^{12}$, se considera que la percepción es un proceso subjetivo mediante el que se realiza una abstracción del mundo, permite al sujeto reconocer elementos, agruparlos en un conjunto y darles sentido y coherencia. El comportamiento humano puede entenderse al analizar las interacciones entre la conducta y la situación.

Se plantea que para Weber la comprensión es importante a fin de entender a las personas, él recomendaba conocer sus experiencias y sus percepciones por medio de la escucha y la observación ${ }^{13}$; denominaba verstehen a la comprensión personal de los motivos y creencias que están detrás de las acciones de las personas ${ }^{14}$. Por ello, resulta interesante indagar en torno a la percepción que el personal de salud tiene respecto al programa DOCC, ya que son los profesionistas quienes guían el comportamiento de la población y definen los resultados del programa. Son pocos los estudios que se interesan por el componente subjetivo en torno a experiencias del personal de salud en su campo profesional, entre ellos se encuentra una investigación enfocada en el significado de la sexualidad en la práctica profesional del personal de salud en formación; en ésta se destaca que el tema de la sexualidad generalmente no se aborda en los planes y programas de estudio, y cuando se hace, se refiere sólo como un aspecto biológico, sin tomar en cuenta los aspectos socioculturales involucrados ${ }^{15}$. No obstante, en los programas de prevención del cáncer cervical subyace el componente sexual. Ante este panorama se justifica la necesidad de identificar los aspectos que contribuyen o limitan la efectividad de las acciones del programa DOCC, especialmente en el área rural, por ser las regiones con mayor tasa de mortalidad por $\mathrm{CaCu}$. Los Centros de Salud seleccionados, para esta investigación, pertenecen a tres jurisdicciones sanitarias del estado, con tasas de mortalidad por $\mathrm{CaCu}$ de 51.8, 15.7 y 13.8 por 10,000 mujeres mayores de 35 años ${ }^{16}$.

El presente artículo muestra una parte de resultados que corresponden a una investigación realizada como tesis doctoral y tiene como propósito conocer cuál es la percepción del personal médico y de enfermería, respecto al desarrollo del programa de detección oportuna de $\mathrm{CaCu}$, específicamente en 
áreas rurales, los hallazgos sientan las bases para dilucidar los motivos que llevan al personal de salud a tomar determinadas acciones que, sin duda, afectan los logros de dicho programa. También, es posible reflexionar desde una perspectiva social, los aspectos ocultos en una subjetividad colectiva, los cuales determinan el perfil de salud o enfermedad de una población.

\section{Métodos}

Para el logro del objetivo, el primer paso fue un acercamiento con las autoridades de los servicios de salud a nivel estatal, de manera especial con los responsables de las áreas de salud reproductiva y del programa DOCC. Posteriormente, se acudió a cada centro de salud para solicitar la participación del personal y establecer fechas y horarios para llevar a cabo las entrevistas.

Participaron en la investigación médicos y enfermeras que prestaron atención directa a las mujeres usuarias del programa DOCC en centros de salud rural, dado que en las áreas rurales hay mayor riesgo de morir por $\mathrm{CaCu}$ que en las zonas urbanas ${ }^{1}$, además de que no se cuenta con las condiciones óptimas para la operatividad del programa: al carecer de recursos humanos, materiales y físicos. Sin dejar de lado que las mujeres que habitan en estas regiones, tienen bajo nivel económico y escolar, iniciaron su vida sexual a temprana edad, tienen altas tasas de fecundidad, así como un restringido acceso a servicios de salud, factores que se consideran de riesgo para padecer o morir por $\mathrm{CaCu}^{17}$. Aunado a ello, la bibliografía deja claro que en este medio, el cáncer cervical no es diagnosticado oportunamente ${ }^{1}$. Para integrar la muestra, se eligió a un centro de salud en cada una de las tres jurisdicciones sanitarias del estado. Se incluyó a personal médico y de enfermería responsables de la ejecución del programa.

El estudio se desarrolló entre los meses de abril y agosto de 2013, en centros de salud rurales que contaran con el programa DOCC y que se ubican en algún municipio que en 2010 registró una tasa de mortalidad por $\mathrm{CaCu}$ mayor a la media estata ${ }^{16}$. El estudio se sometió a la consideración del comité de Ética del programa de Doctorado de la UAM- Xochimilco, el cual dio su aprobación.

La investigadora responsable del proyecto realizó entrevistas semi-estructuradas, mismas que tuvieron una duración promedio de una hora y fueron grabadas en audio (previa autorización de los participantes), para su posterior transcripción. Las personas que aceptaron realizar la entrevista tenían alguna actividad específica en la puesta en marcha del programa DOCC, por lo que participaron dos médicos, que brindan consulta externa, y tres enfermeras, en cada centro de salud. Las enfermeras dijeron "rotar" por los servicios, ya que en ocasiones les corresponde estar en la recepción de la consulta, o bien, en la toma de las muestras del Papanicolaou, o realizar visita domiciliaria. Cabe mencionar que las personas entrevistadas constituían el total de personal de los centros de salud en el turno matutino: No existía ninguna relación de tipo afectivo, laboral ni de ninguna índole entre los participantes y las investigadoras. Para conservar el anonimato de las y los entrevistados, se les asignó una letra (E: Enfermera; M: Médico) y un número para su identificación, sin distinción del centro de salud en el que laboraban. La información se analizó siguiendo los planteamientos de la Teoría Fundamentada con base en la propuesta de Strauss y Corbin ${ }^{18}$. El análisis se desarrolló en tres fases: en la primera, se realizó la codificación abierta mediante la lectura párrafo por párrafo y renglón por renglón, se identificaron los temas que emergieron. En la segunda, codificación axial, a partir de los diferentes códigos se identificaron algunas categorías. Finalmente, en la codificación selectiva, mediante un proceso inductivo, se fueron construyendo los diferentes conceptos a partir de las categorías identificadas. Tanto las entrevistas como su análisis fueron validadas por dos investigadoras y un investigador de la Universidad Autónoma Metropolitana unidad Xochimilco.

\section{Resultados}

Participaron en el estudio 15 profesionales, 13 mujeres y dos hombres con un promedio de edad de 34.3 años, y diferentes niveles académicos. Entre los médicos había personal titulado, que eran los médicos adscritos, así como pasantes de servicio social, que laboran por un año en el centro de salud, 
y cuentan con un apoyo económico que apenas rebasa el salario mínimo. En cuanto al personal de enfermería algunas de las entrevistadas contaban con licenciatura y otras con nivel técnico.

Del análisis de la información de las entrevistas surgió como categoría central la prevención del cáncer, de ésta se derivan varias subcategorías que construyen la percepción que tiene el personal respecto al programa DOCC.

\section{El Papanicolaou como medida preventiva}

El personal de salud, específicamente el personal de enfermería al hablar del programa DOCC, destaca dos aspectos relevantes: el primero, es la simplificación del programa a una sola acción,que es la obtención de muestras cervicales, la cual si bien es la base del programa no constituye su totalidad. El segundo aspecto es la identificación de la prevención del cáncer cervical e incluso otras enfermedades de transmisión sexual son el objetivo del programa. Esta percepción se muestra en las expresiones de las enfermeras.

E1: "Pues es que es un programa (...) que es muy importante que todas las usuarias que inicien su vida sexual activa empiecen a, a realizárselo, es más que nada para prevenir y con los años, ora sí, ¿cómo le dijera? En el transcurso de su vida sexual activa, este, prevenir el cáncer en la mujer. Entonces es muy importante realizarse su Papanicolaou".

E3: "Para evitar, el $\mathrm{CaCu}$ y alguna otra infección, como el virus del papiloma es un cuidado que nos damos a nosotras, para que evitemos esa enfermedad".

Resulta evidente que el personal de salud al hablar del programa DOCC, lo limita a la obtención de muestras cervicales (Papanicolaou), y deja de lado otras acciones incluidas en el programa como son: desarrollar campañas de comunicación educativa para favorecer la adopción de hábitos de vida saludable y la demanda de los servicios para su detección oportuna; mejorar la cobertura y calidad; impulsar la formación y capacitación continua del personal; promover la gestión adecuada de la infraestructura física, equipo y personal; fomentar el acceso, utilización y provisión del diagnóstico y tratamiento integral del $\mathrm{CaCu}$; unificar las acciones con las instituciones del Sistema Nacional de Salud y monitorear y evaluar las acciones de detección; brindar orientación a la población sobre prevención de infecciones de transmisión sexual, identificación de mujeres en riesgo, referencia y contra referencia de mujeres con lesiones a centros de atención médica especializada; desarrollar encuestas sobre la percepción de la población en relación con los factores de riesgo; la prevención y la detección oportuna del CaCu; fomentar la participación de la sociedad civil organizada en la sensibilización y educación sobre el CaCu y el Virus del Papiloma Humano (VPH); diseñar, reproducir y difundir material educativo, comprensible y adecuado para las diferentes audiencias, todas estas, acciones establecidas por el programa nacional de acción específica para la prevención del cáncer cervical ${ }^{1}$. El discurso de las y los entrevistados respecto al desarrollo del programa DOCC, en el sistema de salud, sugiere que perciben la prueba del Papanicolaou como una medida preventiva, esto probablemente porque se ajusta a las características de un programa de prevención; ya que se desarrolla en los centros de salud por personal de enfermería, en el servicio de medicina preventiva previa presentación de la cartilla de la mujer, y en cuyos mensajes promocionales masivos del programa se define a la prueba de Papanicolaou como la acción para prevenir el cáncer cervical. Por otro lado, se destaca que al personal se le evalúa mediante el alcance de metas que dependen de la cobertura lograda, misma que se determina al comparar el número de muestras obtenidas con el total de las mujeres que habitan en la comunidad. Bajo esta lógica el personal de salud entrevistado actúa como lo haría ante cualquier programa de prevención, así como dar prioridad al cumplimiento de metas sin considerar la calidad en la obtención de la muestra, la solicitud y entrega de resultados y el seguimiento de mujeres con lesiones.

\section{Cumplimiento de metas}


En esta subcategoría se hace evidente la preocupación de las y los entrevistados respecto al no cumplimiento de las metas del programa, así como dar preferencia a la repercusión que tendrá para el personal, no obtener el número de muestras que las autoridades sanitarias, a nivel local, les exigen en periodos establecidos, en lugar de interesarse por la detección oportuna de la enfermedad o por evitar muertes prematuras; con ello se evidencia el desconocimiento del propósito principal del programa. La necesidad de cumplir con la meta establecida, se convierte en un aspecto esencial, a tal grado que las enfermeras y los médicos buscan estrategias no incluidas en el programa para lograr obtener el número impuesto de citologías. Una de las acciones a las que recurren consiste en salir a la comunidad, por lo que abandonan la atención en el centro de salud, a buscar a las mujeres en sus domicilios, invitarlas a tomarse la muestra y programarles citas para que acudan al centro de salud, lo cual, además de ser una acción coercitiva, no obedece a la estrategia oportunista marcada en el programa, la cual indica que a la mujer se le invita a realizar un Papanicolaou cuando asiste a medicina preventiva. O bien, otra estrategia que implementan, consiste en trabajar los días de descanso laboral (domingos) para cumplir con el mismo fin. Así, se desvirtúa el propósito del programa DOCC y el compromiso profesional del personal, como se muestra en los relatos de algunas de las enfermeras.

E4: "Cuando ponen metas para hacer Papanicolaous, se sale a la comunidad a invitar, se dirige a las casas, y ya empiezan a citar a las pacientes".

El personal realiza estas acciones aun cuando sabe que las metas no están basadas en datos reales, y que se les exige un número de muestras mayor a la cantidad de población existente en su área de influencia, así lo relata una médica encargada de un centro de salud.

nosotros";

M1: “No sé de dónde las sacan (las metas), a veces son muy elevadas, son muy elevadas, para

Otro médico lo manifiesta de la siguiente manera:

M2: "Inalcanzables, porque pues realmente, no, siento que no es la población realmente la que está, (...) siento que son muy elevadas (...) no es la población real”.

De esta forma, para el personal de salud se desvirtúa el éxito del programa, ya que en lugar de cumplir con el propósito de tomar la muestra para detectar lesiones precancerosas o cancerosas, se ocupan de realizar las citologías cervicales requeridas por las autoridades en un tiempo determinado, si la cifra requerida se rebasa, se valora como un logro del grupo, el relato de una enfermera lo constata:

E5: "Tuvimos mucho éxito porque logramos rebasar el número que tuvimos".

La posibilidad de no obtener una muestra cervical no representa para el personal la pérdida de una oportunidad de hacer un diagnóstico de cáncer, lejos de ello, significa una muestra menos para cumplir la meta. Los siguientes relatos dan cuenta de ello:

E3: "Son oportunidades perdidas para nosotros porque dejamos ir a la paciente y pues sí hay que hacerla regresar (para tomarle la muestra), aunque uno no quiera”.

A pesar de que no existe una forma de castigo o represalia al personal responsable de los centros de salud, el no alcanzar la meta les genera un sentimiento de preocupación e inquietud como lo manifiesta una médica:

M1: “Ahora sí que están con la zozobra de: no cumplimos la meta, con la preocupación, no nos pasa nada pero en nuestra mente no crea”.

Este contexto contribuye también a que el personal se desenvuelva en un ambiente de incomodidad laboral, y en una situación estresante.

Otra de las categorías que emergieron está relacionada con las barreras que el personal de salud percibe para la ejecución del programa DOCC.

Barreras para operar el programa DOCC

Algunas de las limitantes que los y las entrevistadas identifican para que el programa se realice en óptimas condiciones son: la alta demanda de población que solicita atención médica y el poco tiempo 
que el personal dispone para ello; los sentimientos, que en ellos se generan ante un posible diagnóstico de cáncer; la escasez de recursos materiales para la ejecución del programa; los conocimientos que tienen respecto al programa; la habilidad en el manejo de pacientes y las condiciones laborales del personal de salud.

\section{Alta demanda}

Los profesionales entrevistados reconocen que existen limitantes para la praxis del programa; una de estas se identifica con la alta demanda de pacientes que se tienen que atender en el horario de consulta, lo cual impide hacer todas las recomendaciones en cuanto a la prevención, al respecto una médica en servicio social menciona:

M4: "Tienes que hacer la sugerencia de que se haga el Papanicolaou, la sugerencia de que se haga exploración de mama, la sugerencia de que darle una orientación sobre planificación familiar, de una orientación nutricional etc., etc., etc. y que haga ejercicio y en teoría pues tenemos 20 minutos (...) tienes una saturación increíble de pacientes”.

\section{Sentimientos de temor y compasión}

Un aspecto relevante lo constituye la forma en que se brinda la información a las mujeres cuyo resultado de la citología es anormal, lo que ocasiona en el personal sentimientos de compasión hacia la mujer y temor de informarle sobre su resultado de forma inadecuada, que le cause conflicto o tristeza, así se deduce en el siguiente fragmento:

M5: “Decirle a alguien “tienes cáncer”, o “tienes una lesión que se puede hacer cáncer”, pues sí, los impacta pero si uno les explica los cuidados que deben de tener (...) me tocó, que sí, el doctor sí le explicó (a una paciente) bien a consciencia que pues no era una lesión maligna, pero que debía de tener cuidado por esto, y esto y esto, igual tal vez lo aceptó, en el momento pues no le quedaba de otra”.

También, se identificó en las entrevistas que el personal médico y de enfermería experimentan sentimientos de temor cuando se le plantea, la posibilidad de que él o algún familiar cercano pudieran padecer cáncer. Sin embargo, cuando la situación hipotética se refiere a las pacientes, ellos refieren sentir compasión por ellas. Los pensamientos de fatalidad, ligados al diagnóstico de cáncer generan angustia en el personal cuando el resultado de la prueba es positivo a $\mathrm{CaCu}$, como lo muestra la expresión de una enfermera:

E4: “...jay no!, sería algo muy fuerte, ay, no sé, no Dios mío, no sabría ni qué decirle, no pues (...) desde que es NIC I, porque con el simple hecho de que sale que es NIC I es, para nosotros, bueno al menos en mi persona, feo, porque decimos que hay algo más ¿no? (...) como que, pues sí siento una sensación ¡uy! que, como miedo”.

\section{Recursos materiales}

El personal de salud identifica como una limitante para el desarrollo del programa: la escasez de recursos materiales, aunado a la dificultad para conseguirlos en las comunidades rurales, esta situación genera en el personal sentimientos de frustración, impotencia, más aún, cuando su intento de solventar las carencias se torna imposible:

M4: "Se dio una ocasión en la que no se tomaron Papanicolaous porque no había laminillas ni espátulas, entonces, tuvieron que mandar a las señoras a comprar una sola (señalando al levantar un dedo), un portaobjetos, entonces, dígame quién, una señora de pueblito iba a saber, para empezar, qué era un portaobjetos, no son cosas que encuentres en cualquier tienda por ejemplo (...) no hay insumos suficientes". 
El conocimiento que tiene el personal médico y de enfermería sobre los objetivos, estrategias y líneas de acción del programa es insuficiente, en ocasiones se tiene completo desconocimiento de éste, del mismo modo, es limitada la información con que cuentan sobre la fisiopatología de la enfermedad, como lo expresa un médico en servicio social:

M5: "No, la verdad no (desconoce el programa DOCC). Le digo, como llevo cuatro días. mmm-mmm (negando con la cabeza). Este... mire, no, la verdad le seré sincero, llevo cuatro días aquí, y no pues si acaso ahorita porque me, me comentó de lo que significa, pero no, no. ¿Cómo dice que se llama? (risas), a ver deje hacer memoria... más o menos, le voy a ser sincero, más o menos. Sí, sé que, obviamente, igual, ya casi me exhibí, tengo que obviamente leer más, pero sí es, tengo el conocimiento de que, este, existen obviamente los grados de malignidad, NIC I, NIC II, NIC III o lesión, es que también depende de la clasificación.

Al explorar sobre los cursos de capacitación que recibe el personal, se detecta que estos son percibidos como un premio o un privilegio, se muestran deseosos de recibir una invitación para asistir a algún curso de actualización, como lo muestra el siguiente fragmento:

E2: "Que... yo donde me conviden, me oferten, yo estoy ahora sí que muy dispuesta, a mí me encantan las actualizaciones, ajá, y eso es lo que nos hace falta casi a la mayoría”.

El personal de enfermería refiere experiencias estresantes en la obtención de muestras cervicales, por lo que necesita capacitación técnica, para adquirir habilidad y destreza, así lo expresa una enfermera:

E1: "Pues sobre esto ¿no?, de la, lo que es este, la toma de Papanicolaou, porque yo me he encontrado... ¡ay! (suspira), haga de cuenta que, pones el espejo, llegas al cuello, al momento de quitarlo no sale, como que hace un vacío, yo no sabía que pasaba eso (...) Entonces yo decía “¡Dios mío!, ¿Y ahora qué hago, no?”, ¿no?, tratas de jalarlo y se te viene todo el cuello, se te viene...”.

Estas experiencias son duros ejemplos de la falta de habilidades del personal; y muestras claras del riesgo en el que están las mujeres al ser atendidas por este personal.

\section{Condiciones de trabajo}

La rotación y cambios de personal de salud afectan tanto a las usuarias del servicio como al mismo personal. Las y los entrevistados destacan que en las usuarias, esto genera una sensación de desconfianza que expresan de diversas maneras. Por otra parte, entre las enfermeras se manifiesta un sentimiento de incomodidad y rechazo hacia las usuarias del servicio, por sentir que estas cuestionan sus conocimientos y habilidades profesionales, ello tiene como consecuencia que el personal de enfermería muestre una actitud impositiva, al hacer saber que ellas son las que permanecerán desde ese momento en el centro de salud.

La siguiente experiencia de una enfermera lo muestra:

E2: “Cuando llegué aquí me decían ¿y Mary? (era la que estaba aquí, la enfermera María) “¿y Mary, no va a venir Mary? ¿Ahora le tocó, ahora usted la cubrió?”, "No, no la cubrí, ya me voy a quedar por un año por el momento aquí".

Por su parte, el personal médico, tiende a permanecer en los centros de salud por periodos cortos y variables, situación que les genera incertidumbre debido a la inestabilidad laboral, tal como lo refleja en siguiente párrafo:

M6: "Cada año (firman mi contrato)...Sí. Bueno, últimamente ya cada seis meses. Porque antes era por año ahora cada seis meses... No sé, porqué, no nos han explicado nada. No hay garantía”.

\section{Discusión}

Los estudios para evaluar la efectividad del programa de acción específico de Prevención y control del cáncer de la mujer, generalmente no consideran al personal de salud responsable de su ejecución, 
cuando ha sido abordado, es desde un punto de vista gerencial, por lo cual concluye que el limitado impacto en la tasa de mortalidad por cáncer cervical se debe al bajo nivel de conocimiento del personal. Otra de las formas para intentar explicar los resultados del programa, es siguiendo los planteamientos del modelo médico hegemónico caracterizado por el predominio del enfoque biológico, e individualista que hace al sujeto responsable de su salud y su cuidado ${ }^{19}$, en el caso de las mujeres, se les deposita toda la responsabilidad de no asistir a revisiones al programa DOCC. Desde esta perspectiva, algunos estudios señalan que las mujeres perciben el Papanicolaou como una medida preventiva ${ }^{20,21}$, en otras investigaciones se identifica que el conocimiento sobre el VPH es un factor importante para que las mujeres acudan al programa $\mathrm{DOCC}^{22,23}$. En otras investigaciones se ha integrado al personal de salud, pero desde un punto de vista evaluativo9, por lo cual se define que la poca efectividad del programa se debe al bajo nivel de conocimientos del personal o bien por carecer de recursos materiales para operar el programa ${ }^{10}$.

En esta investigación se manifestó que la forma en que el personal percibe el programa DOCC está definida por la imposición de metas en la obtención de muestras cervicales, los sentimientos de temor y compasión que experimenta el personal de salud, la deficiencia de recursos materiales; sus conocimientos sobre el programa y sus condiciones laborales. Estas situaciones ocasionan que el personal transforme la realidad según su perspectiva, misma que depende de su ubicación, y su contexto histórico social y cultural. La percepción resultante del conflicto dialéctico entre una proyección institucional, en la que fue creado el programa, y la transformación de esta proyección a la realidad a la que se enfrentan, sin dejar de lado la propia realidad individual.

Es importante considerar que muchos de los aspectos percibidos, en esta investigación, tienen su base en la formación académica, por ello es indispensable hacer que en los profesionales de enfermería en formación hagan énfasis en el correcto uso de los términos. Hacer muy clara la diferencia entre detección y prevención de enfermedades, ya que el personal de salud insiste en la prevención, pero no identifica el tipo de prevención ni el momento en que se debe promover, esto genera confusión entre el personal, asimismo se trasmite información errónea a la población; ello se evidencia en el intento del personal de salud de convencer a las mujeres de acceder a realizarse el Papanicolaou, promueven que esta prueba es útil (y casi indispensable) para "prevenir" el $\mathrm{CaCu}$, sin señalar que es sólo el primer paso para la obtención de un diagnóstico de la enfermedad. Ante esta situación, no sólo se pone en riesgo la efectividad del programa, sino también, y lo más relevante, la salud de la población y el bienestar de los mismos profesionales.

Se deduce entonces que el objetivo del programa, su esencia no está clara para el personal de salud ni para la población usuaria. El programa DOCC es mucho más que sólo el hecho de tomar Papanicolaous, incluye acciones a nivel poblacional de información, educación, acciones comunitarias, identificación de mujeres en riesgo y otras acciones. Sólo las actividades que involucran la educación para la salud y la información podrían considerarse como acciones preventivas.

Así, el personal considera que ha concluido con el propósito del programa al obtener la muestra de Papanicolaou y cumplir con la meta impuesta, por lo tanto se hace caso omiso de la promoción de la salud, seguimiento y control de las mujeres con lesiones cervicales, lo que repercute en una ausencia de tratamiento oportuno y por ende, una elevada mortalidad por esta causa.

Un aspecto que valdría la pena considerar, en la asignación del programa, es que el procedimiento de la obtención de muestras cervicales involucra además, la exploración física y visual de genitales internos y externos de la mujer. Por lo que cuando se realiza por personal médico, le es posible detectar signos de enfermedades de transmisión sexual y alteraciones en los órganos sexuales, tales como secreciones, úlceras, lesiones, presencia de verrugas o condilomas, sangrados anormales y otros que no se identifican de otra forma. En este caso, es el personal de enfermería quien obtiene la muestra, la mayoría de las veces sin capacitación sobre la obtención de muestras y menos aún sobre la inspección visual, por lo que no se logra un diagnóstico integral que conlleve a un tratamiento. 
Es probable que el desarrollo de esta investigación por ser en el medio rural influya en los resultados, ya que en este medio, como lo muestra García, existen inequidades en la puesta en marcha del programa en esta población ${ }^{24}$, la dotación de insumos es irregular, las opciones de actualización profesional dirigidas al personal de salud son mínimas, estas exigen traslados a otras ciudades o localidades, y la posibilidad de que el personal de salud obtenga una plaza laboral definitiva es muy baja.

\section{Conclusiones}

Desde la salud colectiva, el proceso salud-enfermedad, como fenómeno social, resulta de la compleja e intrincada relación entre una base biológica y factores socioeconómicos, culturales, psicológicos y conductuales, que surgen a nivel individual, para influirlo o modificarlo; por lo que no puede considerarse ni concreto ni generalizable. Es preciso comprender, en el proceso salud-enfermedad, la percepción de los fenómenos relacionados tanto en los proveedores como en los receptores de los servicios de salud. Así como la forma en que estas percepciones afectan las acciones a favor de la salud colectiva para cada sujeto y lugar.

En esta investigación se evidencia que el personal de salud percibe al programa DOCC como la estrategia para obtener muestras cervicales, que tienen como fin la prevención del cáncer cervical e incluso otras enfermedades de transmisión sexual; se pone especial énfasis en el logro de las metas establecidas para su centro de salud; aun cuando se sabe que estas exigencias no corresponden con la población que hay en su localidad. Dichas acciones los alejan de uno de sus principales objetivos, la salud de la población, sea individual o en colectivo.

En la narrativa del personal de enfermería se refleja la lógica del pensamiento social, que proclama que prevenir las enfermedades es prioritario y asume que ésta es su responsabilidad. No obstante, este genuino interés, existen barreras de conocimiento que limitan sus propósitos.

El dominio de la técnica para la obtención de muestras cervicales lo obtiene el personal mediante la ejecución repetida del procedimiento, sin capacitación formal.

Las oportunidades de capacitación para el personal de salud son limitadas, ya que estás están dirigidas principalmente al personal administrativo, quienes no son los principales ejecutores en el programa DOCC. Cuando hay alguna oportunidad de capacitación, el personal médico y de enfermería advierte que la asistencia a los cursos existentes es un privilegio, al que acuden por invitación, por lo cual asumen una actitud pasiva, esperan que les llegue una invitación para ser actualizados profesionalmente.

En esta investigación se evidencia que el personal carece de información sobre el programa DOCC. De igual forma, emergen una serie de limitantes para su operación, sin dejar de lado la inseguridad laboral que enfrenta el personal de salud entrevistado. Esta situación genera una serie de sentimientos, frustración, impotencia, enojo y tristeza que impactan no sólo su desarrollo personal y profesional, sino que llevan a la población a no confiar en el programa. El desconocimiento de la forma correcta de realizar los procedimientos causa en los profesionales inseguridad, incertidumbre y temor, lo que afecta el desempeño de su profesión, por lo cual limita las acciones de los profesionales y orientándolos a la obtención de objetivos distintos a los establecidos.

Por tanto, resulta relevante tomar en cuenta la información obtenida en esta investigación, específicamente para reforzar los planes y programas de estudio de las carreras de enfermería y medicina, en cuanto al conocimiento de la prevención del $\mathrm{CaCu}$; y su detección oportuna; así como una diferenciación clara entre estas dos acciones. Es indispensable que en la formación académica se realicen esfuerzos para que los estudiantes obtengan conocimientos, desarrollen habilidades y adquieran competencias que les permitan un comportamiento profesional basado en conocimientos científicos, generar en los estudiantes el correcto uso de términos médicos, la lectura crítica de programas de salud, la búsqueda de información en medios impresos o digitales, así como el interés por la actualización y el crecimiento profesional. 


\section{Responsabilidades éticas}

Protección de personas y animales. Los autores declaran que para esta investigación no se han realizado experimentos en seres humanos ni en animales.

Confidencialidad de los datos. Los autores declaran que en este artículo no aparecen nombres que permitan identificar a los participantes.

Derecho a la privacidad y consentimiento informado. Los autores han obtenido el consentimiento informado de los participantes. Este documento obra en poder del autor de correspondencia.

Financiamiento. Ninguno

Conflicto de intereses. Los autores declaran no tener ningún conflicto de intereses.

\section{Referencias}

1. Palacio-Mejía LS, Lazcano-Ponce E, Allen-Leigh B, et al. Regional differences in breast and cervical cancer mortality in Mexico between 1979-2006. Salud pública Mex. 2009; 51(2): s208-s219.

2. Secretaría de Salud. NOM-014-SSA2-1994, Para la prevención, tratamiento y control de cáncer del cuello del útero y de la mama en la atención primaria. México: SSA; 1994.

3. Kocjan BJ, Bzhalava D, Forslund O, et al. Molecular methods for identification and characterization of novel papillomaviruses. Clin Microbiol Infect. 2015; 21(9): 808-16.

https://doi.org/10.1016/j.cmi.2015.05.011

4. Van Doorslaer K, Li Z, Xirazagar S, et al. The Papillomavirus Episteme: a major update to the papillomavirus sequence database. Nucleic Acids Res. 2017; 45(D1): D499-D506.

https://doi.org/10.1093/nar/gkw879

5. Lazcano, E. El cáncer cervical: nuevas perspectivas de prevención y control. Rev Per Ginecol Obstet. 2007; 53(2): 110-8.

6. Organización Panamericana de la Salud. Programa de Cáncer. Cáncer cervicouterino. Washington: OPS; 2016. [consultado 12 abril 2017] Disponible en: https://bit.ly/2s4OCXr

7. Secretaría de Salud. Estadísticas de Cáncer de Mama y Cáncer Cérvico Uterino. México: SSA. Dirección General de Información en Salud; 2013. [consultado 12 abril 2017]. Disponible en: https://bit.ly/2GXSoZT

8. Hernández-Hernández DM, Apresa-García T, Patlán-Pérez RM. Panorama epidemiológico del cáncer cervicouterino. Rev. Med. Inst. Mex. Seguro Soc. 2015; 53(Supl2): 154-61.

9. Arillo-Santillan E, Nigenda G, Sánchez-Prado VM, et al. México city physicians' awareness about cervical cancer prevention: implications for cancer screening. J Cancer Educ. 2001; 16(2): 75-9. https://doi.org/10.1080/08858190109528736

10. Bronfman M, Castro R, Zúñiga E, et al. "Hacemos lo que podemos": los prestadores de servicios frente al problema de la utilización. Salud pública Mex. 1997; 39(6): 546-53.

11. Chavira Jj. Programa de Mejora: Cruzada por la Calidad de los Servicios de Salud. La Percepción del Personal Médico en un Hospital Público. Reflexiones desde los Estudios Críticos de gestión. [Tesis doctoral]. CDMX: UAM; 2010.

12. Arnheim R. Arte y percepción visual. Madrid: Alianza Editorial; 1986.

13. Grbich C. Qualitative Research in Health. An Introduccion. London: Sage Publications; 1999.

14. Taylor SJ, Bogdan R. Introducción a los métodos cualitativos de investigación. $3^{\text {a }}$ ed. Buenos Aires: Paidós Básica; 2000.

15. Salinas AA, Jarillo EC. La confrontación de la sexualidad en la práctica profesional de los futuros médicos: la mirada de los pasantes de medicina. Cien Saude Colet 2013; 18(3): 733-42.

http://dx.doi.org/10.1590/S1413-81232013000300019

16. Secretaría de Salud. Morelos. Mortalidad 2010: Aspectos Relevantes. Boletín Estadístico Núm. 14. Morelos: SSA; 2012. 
17. Rosell E, Muñoz A, Cepero F, et al. Factores de riesgo del cáncer de cuello uterino. Archivo médico de Camagüey. 2007; 11(1): 1-9.

18. San Martín D. Teoría fundamentada y Atlas.ti: recursos metodológicos para la investigación educativa. Rev. electrón. de investig. educ. 2014; 16(1): 104-22.

19. Menéndez EL. Modelo Médico Hegemónico y Atención Primaria. Segundas Jornadas de Atención Primaria de la Salud. Buenos Aires: CONAMER; 1988. P.451-64.

20. Urrutia MT, Poupin L. Las mujeres con cáncer de cuello uterino: percepciones acerca de la prueba de Papanicolaou. Aquichan 2015; 15(4): 499-507. https://doi.org/10.5294/aqui.2015.15.4.5

21. Urrutia MT, Araya A, Poupin L. ¿Por qué las mujeres no se toman el Papanicolaou? Respuestas entregadas por los profesionales del programa cáncer cervicouterino - Auge del servicio de salud metropolitano sur oriente. Rev. chil. obstet. ginecol. 2010; 75(5): 284-9.

http://dx.doi.org/10.4067/S0717-75262010000500002

22. Hernández-Márquez CI, Brito-García I, Mendoza-Martínez M, et al. Conocimiento y creencias de mujeres del estado de Morelos sobre el virus del papiloma humano. Rev.cuba.enferm. 2016; 32(4).

23. Ybarra JL, Pérez BE, Romero D. Conocimiento y creencias sobre la prueba de Papanicolaou en estudiantes universitarios. Psicología y salud. 2012; 22(2): 185-94.

24. García-Pérez H, Merino M. La toma de la muestra de Papanicolaou en población indígena migrante en el noroeste de México: el caso del programa “Dile a una amiga”. Salud pública Méx. 2015; 57(1): 1-2. 